\title{
Jordan Curve Theorems with Respect to Certain Pretopologies on $\mathbb{Z}^{2}$
}

\author{
Josef Šlapal \\ Brno University of Technology, Department of Mathematics, \\ 61669 Brno, Czech Republic \\ slapal@fme.vutbr.cz \\ http://at.yorku.ca/h/a/a/a/10.htm
}

\begin{abstract}
We discuss four quotient pretopologies of a certain basic topology on $\mathbb{Z}^{2}$. Three of them are even topologies and include the wellknown Khalimsky and Marcus-Wyse topologies. Some known Jordan curves in the basic topology are used to prove Jordan curve theorems that identify Jordan curves among simple closed ones in each of the four quotient pretopologies.
\end{abstract}

\section{Introduction}

When studying geometric and topological properties of (two-dimensional) digital images, we need the digital plane $\mathbb{Z}^{2}$ to be provided with a convenient structure. Here, the convenience means that such a structure satisfies some analogues of basic geometric and topological properties of the Euclidean topology on $\mathbb{R}^{2}$. For example, it is usually required that an analogue of the Jordan curve theorem be valid. (Recall that the classical Jordan curve theorem states that any simple closed curve in the Euclidean plane separates this plane into exactly two components). In the classical approach to this problem (see e.g. [11] and [12]), graph theoretic tools were used for structuring $\mathbb{Z}^{2}$, namely the well-known binary relations of 4-adjacency and 8-adjacency. Unfortunately, neither 4-adjacency nor 8-adjacency itself allows an analogue of the Jordan curve theorem - cf. [8]. To eliminate this deficiency, a combination of the two binary relations has to be used. Despite this inconvenience, the graph-theoretic approach was used to solve many problems of digital image processing and to create useful graphic software. In [5], a new, purely topological approach to the problem has been proposed which utilizes a convenient topology on $\mathbb{Z}^{2}$, called Khalimsky topology (cf. [4]), for structuring the digital plane. At present, this topology is one of the most important concepts of the theory called digital topology. It has been studied and used by many authors, e.g., [2] and [6]-[9]. The possibility of structuring $\mathbb{Z}^{2}$ by using closure operators more general than the Kuratowski ones is discussed in [13] and [14].

In [15], a new, convenient topology on $\mathbb{Z}^{2}$ has been introduced and studied. It was shown there that this topology has some advantages over the Khalimsky one. The new topology was further investigated in [16] where it was shown that 
its quotient topologies include the Khalimsky topology as well as two other convenient topologies on $\mathbb{Z}^{2}$. A certain quotient pretopology of the new topology was also studied in [16]. In the present note we continue the study from [16] and prove a Jordan curve theorem for each of the three quotient topologies and also for the quotient pretopology from [16].

\section{Preliminaries}

For the topological terminology used we refer to [3]. Throughout the note, all topologies dealt with are thought of as being (given by) Kuratowski closure operators. Thus, a topology on a set $X$ is a map $p$ : exp $X \rightarrow \exp X$ (where exp $X$ denotes the power set of $X$ ) fulfilling the following four axioms:

(i) $p \emptyset=\emptyset$,

(ii) $A \subseteq p A$ for all $A \subseteq X$,

(iii) $p(A \cup B)=p A \cup p B$ for all $A, B \subseteq X$,

(iv) $p p A=p A$ for all $A \subseteq X$.

The pair $(X, p)$ is then called a topological space. If $p$ satisfies (i)-(iii) but not necessarily (iv), then it is called a pretopology and $(X, p)$ is called a pretopological space. The topological concepts used for pretopologies in this note are obtained by natural extensions of these concepts defined for topologies. In the literature, pretopologies are also known as Cech closure spaces - cf. [1].

A map $f:(X, p) \rightarrow(Y, q)$ between topological (or pretopological, respectively) spaces $(X, p)$ and $(Y, q)$ is said to be continuous if $f(p A) \subseteq q(f(A))$ whenever $A \subseteq X$ - cf. [3] (or [1], respectively).

As usual, given a topological space $(X, p)$ and a surjection $e: X \rightarrow Y$, a topology (pretopology) $q$ on $Y$ is called the quotient topology (quotient pretopology) of $p$ generated by $e$ if $q$ is the finest topology (pretopology) on $Y$ for which $e:(X, p) \rightarrow(Y, q)$ is continuous. Here, as usual, given topologies (pretopologies) $q$ and $r$ on $X, q$ is said to be finer than $r$ (and $r$ coarser than $q$ ) if $q A \subseteq r A$ for every $A \subseteq X$.

We will need the following lemma resulting from [14], Corollary 1.5:

Lemma 1. Let $(X, p)$ be a pretopological space, $e: X \rightarrow Y$ be a surjection and let $q$ be the quotient pretopology of $p$ on $\mathbb{Z}^{2}$ generated by e. Let e have the property that $e^{-1}(\{y\})$ is connected in $(X, p)$ for every point $y \in Y$ and let $B \subseteq Y$ be a subset. Then $B$ is connected in $(Y, q)$ if and only if $e^{-1}(B)$ is connected in $(X, p)$.

Let us note that, for a topological space $(X, p)$ and a quotient topology of $p$, the statement of the previous Lemma need not be true. It is true if, for example, $B$ is closed or open in $(Y, q)$.

Recall that the connectedness graph of a pretopology $p$ on $X$ is the undirected simple graph whose vertex set is $X$ and in which a pair of vertices $x, y$ is adjacent (i.e., joined by an edge) if and only if $x \neq y$ and $\{x, y\}$ is a connected subset of $(X, p)$. A pretopology $p$ on $X$ is called an Alexandroff pretopology if 
$p A=\bigcup_{x \in A} p\{x\}$ for every $A \subseteq X$ and it is called a $T_{0}$-pretopology if $x \in p\{y\}$ and $y \in p\{x\}$ imply $x=y$ whenever $x, y \in X$. So, if $p$ is an Alexandroff pretopology, then it is given by determining closures of all points of $\mathbb{Z}^{2}$. Thus, $p$ is then also given by its connectedness graph provided that every edge of the graph is adjacent to a point which is known to be closed or to a point which is known to be open (in which case $p$ is $T_{0}$ ). Therefore, in all the (connectedness) graphs displayed, the closed points are ringed while the mixed ones (i.e., the points that are neither closed nor open) are boxed. Thus, the points that are neither ringed nor boxed are open (note that no points may be both closed and open in a connected space with more than one point).

By a (discrete) simple closed curve in a pretopological space $(X, p)$ we mean, in accordance with [16], a nonempty, finite and connected subset $C \subseteq X$ such that, for each point $x \in C$, there are exactly two points of $C$ adjacent to $x$ in the connectedness graph of $p$. A simple closed curve $C$ in $(X, p)$ is said to be a (discrete) Jordan curve if it separates $(X, p)$ into precisely two components (i.e., if the subspace $X-C$ of $(X, p)$ consists of precisely two components). In the remaining part of the paper, pretopologies on $\mathbb{Z}^{2}$ only will be dealt with.

Let $z=(x, y) \in \mathbb{Z}^{2}$ be a point. We put

$$
\begin{aligned}
& H_{2}(z)=\{(x+k, y) ; k \in\{-1,0,1\}\}, \\
& V_{2}(z)=\{(x, y+l) ; l \in\{-1,0,1\}\}, \\
& D_{4}(z)=H_{2}(z) \cup\{(x-1, y-1),(x+1, y-1)\}, \\
& U_{4}(z)=H_{2}(z) \cup\{(x-1, y+1),(x+1, y+1)\}, \\
& L_{4}(z)=V_{2}(z) \cup\{(x-1, y-1),(x-1, y+1)\}, \\
& R_{4}(z)=V_{2}(z) \cup\{(x+1, y-1),(x+1, y+1)\} .
\end{aligned}
$$

Next, we put

$$
\begin{aligned}
& A_{4}(z)=H_{2}(z) \cup V_{2}(z), \\
& A_{8}(z)=H_{2}(z) \cup L_{4}(z) \cup R_{4}(z)\left(=V_{2}(z) \cup D_{4}(z) \cup U_{4}(z)\right), \text { and } \\
& A_{4}^{\prime}(z)=\{z\} \cup\left(A_{8}(z)-A_{4}(z)\right) .
\end{aligned}
$$

Thus, each of the sets introduced above consists of the point $z$ and some other points whose number equals the index of the symbol denoting this set. In the literature, the points of $A_{4}(z)$ and $A_{8}(z)$ different from $z$ are said to be 4 adjacent and 8-adjacent to $z$, respectively. It is natural to call the points, different from $z$, of $H_{2}(z), V_{2}(z), D_{4}(z), U_{4}(z), L_{4}(z), R_{4}(z)$ and $A_{4}^{\prime}(z)$ horizontally 2adjacent, vertically 2-adjacent, down 4-adjacent, up 4-adjacent, left 4-adjacent, right 4-adjacent and diagonally 4-adjacent to $z$, respectively. Clearly, each of these adjacencies implies 8-adjacency.

\section{Topology $w$ and Some of Its Quotients}

In this section, we repeat some results from [16] (without proofs) that will be needed in the sequel. 
We denote by $w$ the Alexandroff topology on $\mathbb{Z}^{2}$ given as follows:

For any point $z=(x, y) \in \mathbb{Z}^{2}$,

$$
w\{z\}=\left\{\begin{array}{l}
A_{8}(z) \text { if } x=4 k, y=4 l, k, l \in \mathbb{Z}, \\
A_{4}^{\prime}(z) \text { if } x=2+4 k, y=2+4 l, k, l \in \mathbb{Z}, \\
D_{4}(z) \text { if } x=2+4 k, y=1+4 l, k, l \in \mathbb{Z}, \\
U_{4}(z) \text { if } x=2+4 k, y=3+4 l, k, l \in \mathbb{Z}, \\
L_{4}(z) \text { if } x=1+4 k, y=2+4 l, k, l \in \mathbb{Z}, \\
R_{4}(z) \text { if } x=3+4 k, y=2+4 l, k, l \in \mathbb{Z}, \\
H_{2}(z) \text { if } x=2+4 k, y=4 l, k, l \in \mathbb{Z}, \\
V_{2}(z) \text { if } x=4 k, y=2+4 l, k, l \in \mathbb{Z}, \\
\{z\} \text { otherwise. }
\end{array}\right.
$$

Clearly, $w$ is connected and $T_{0}$. A portion of the connectedness graph of $w$ is shown in the following figure:

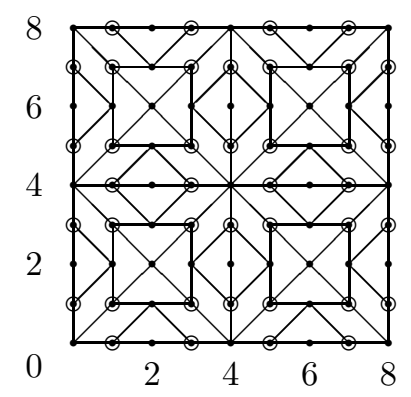

Recall [5] that the Khalimsky topology on $\mathbb{Z}^{2}$ is the Alexandroff topology $t$ given as follows:

For any $z=(x, y) \in \mathbb{Z}^{2}$,

$$
t\{z\}=\left\{\begin{array}{l}
A_{8}(z) \text { if } x, y \text { are even } \\
H_{2}(z) \text { if } x \text { is even and } y \text { is odd } \\
V_{2}(z) \text { if } x \text { is odd and } y \text { is even } \\
\{z\} \text { otherwise. }
\end{array}\right.
$$

The Khalimsky topology is connected and $T_{0}$; a portion of its connectedness graph is shown in the following figure.

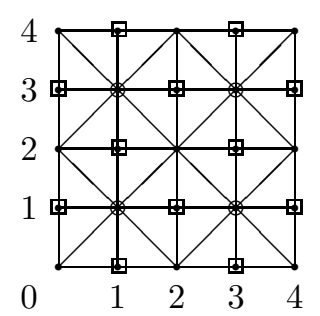


Theorem 1. The Khalimsky topology is the quotient topology of $w$ generated by the surjection $f: \mathbb{Z}^{2} \rightarrow \mathbb{Z}^{2}$ given as follows:

$$
f(x, y)=\left\{\begin{array}{l}
(2 k, 2 l) \text { if }(x, y)=(4 k, 4 l), k, l \in \mathbb{Z}, \\
(2 k, 2 l+1) \text { if }(x, y) \in A_{4}(4 k, 4 l+2), k, l \in \mathbb{Z}, \\
(2 k+1,2 l) \text { if }(x, y) \in A_{4}(4 k+2,4 l), k, l \in \mathbb{Z}, \\
(2 k+1,2 l+1) \text { if }(x, y) \in A_{4}^{\prime}(4 k+2,4 l+2), k, l \in \mathbb{Z} .
\end{array}\right.
$$

Another well-known topology on $\mathbb{Z}^{2}$ is the Marcus-Wyse one (cf. [10]), i.e., the Alexandroff topology $s$ on $\mathbb{Z}^{2}$ given as follows:

For any $z=(x, y) \in \mathbb{Z}^{2}$,

$$
s\{z\}=\left\{\begin{array}{l}
A_{4}(z) \text { if } x+y \text { is odd } \\
\{z\} \text { otherwise }
\end{array}\right.
$$

The Marcus-Wyse topology is connected and $T_{0}$, too. A portion of its connectedness graph is shown in the following figure.

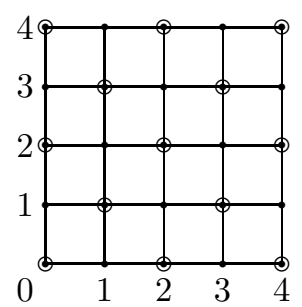

Theorem 2. The Marcus-Wyse topology is the quotient topology of $w$ generated by the surjection $g: \mathbb{Z}^{2} \rightarrow \mathbb{Z}^{2}$ given as follows:

$$
g(x, y)=\left\{\begin{array}{c}
(k+l, l-k) \text { if }(x, y) \in A_{8}(4 k, 4 l), k, l \in \mathbb{Z}, \\
(k+l+1, l-k) \text { if }(x, y)=(4 k+2,4 l+2) \text { for some } k, l \in \mathbb{Z} \\
\text { with } k+l \text { odd or }(x, y) \in A_{12}(4 k+2,4 l+2) \text { for some } \\
k, l \in \mathbb{Z} \text { with } k+l \text { even. }
\end{array}\right.
$$

Let $v$ be the Alexandroff topology on $\mathbb{Z}^{2}$ given as follows:

For any $z=(x, y) \in \mathbb{Z}^{2}$,

$$
v\{z\}=\left\{\begin{array}{l}
H_{2}(z) \text { if } x \text { is odd and } y \text { is even } \\
V_{2}(z) \text { if } x \text { is even and } y \text { is odd } \\
A_{4}^{\prime}(z) \text { if } x, y \text { are odd } \\
\{z\} \text { if } x, y \text { are even. }
\end{array}\right.
$$


Evidently, $v$ is connected and $T_{0}$. A a portion of its connectedness graph is shown in the following figure:

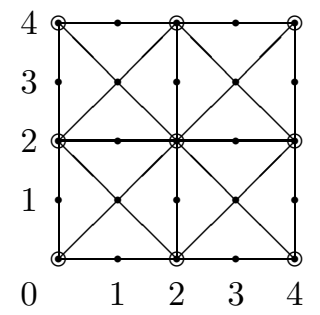

Theorem 3. $v$ is the quotient topology of $w$ generated by the surjection $h: \mathbb{Z}^{2} \rightarrow$ $\mathbb{Z}^{2}$ given as follows:

$$
h(x, y)=\left\{\begin{array}{l}
(2 k, 2 l) \text { if }(x, y) \in A_{8}(4 k, 4 l), k, l \in \mathbb{Z}, \\
(2 k, 2 l+1) \text { if }(x, y) \in H_{2}(4 k, 4 l+2), k, l \in \mathbb{Z}, \\
(2 k+1,2 l) \text { if }(x, y) \in V_{2}(4 k+2,4 l), k, l \in \mathbb{Z}, \\
(2 k+1,2 l+1) \text { if }(x, y)=(4 k+2,4 l+2), k, l \in \mathbb{Z} .
\end{array}\right.
$$

Let $u$ be the Alexandroff pretopology on $\mathbb{Z}^{2}$ defined as follows:

For any point $z=(x, y) \in \mathbb{Z}^{2}$,

$$
u\{z\}=\left\{\begin{array}{l}
A_{4}(z) \text { if both } x \text { and } y \text { are odd or }(x, y)=(4 k+2 l, 2 l+2), k, l \in \mathbb{Z}, \\
A_{8}(z) \text { if }(x, y)=(4 k+2 l, 2 l), k, l \in \mathbb{Z}, \\
\{z\} \text { otherwise. }
\end{array}\right.
$$

Then $u$ is connected and $T_{0}$ with a portion of its connectedness graph shown in the following figure:

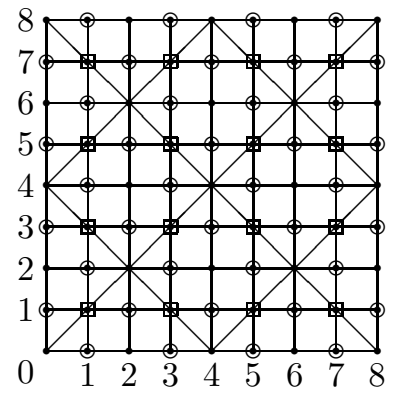

Theorem 4. $u$ is the quotient pretopology of $w$ generated by the surjection $d$ : $\mathbb{Z}^{2} \rightarrow \mathbb{Z}^{2}$ given as follows:

$$
d(x, y)=\left\{\begin{array}{l}
(2 k+2 l+1,2 l-2 k+1) \text { if }(x, y) \in A_{4}(4 k, 4 l+2), k, l \in \mathbb{Z}, \\
(2 k+2 l+1,2 l-2 k-1) \text { if }(x, y) \in A_{4}(4 k+2,4 l), k, l \in \mathbb{Z}, \\
\left(\frac{x+y}{2}, \frac{y-x}{2}\right) \text { if } x, y \text { are odd or }(x, y)=(4 k+2 l, 2 l), k, l \in \mathbb{Z} .
\end{array}\right.
$$




\section{Jordan Curve Theorems}

It can easily be seen that the Khalimsky topology, the Marcus-Wyse topology and the topology $v$ are not only quotient topologies but also quotient pretopologies of $w$ (generated by $f, g$ and $h$, respectively). It is also evident that the inverse image of a singleton under any of the four mappings $f, g, h$ and $d$ is a connected subset of $\left(\mathbb{Z}^{2}, w\right)$. Thus, the assumptions of Lemma 1 are satisfied if $p=w, q \in\{t, s, v, u\}$ and $e$ is the respective surjection $f, g, h$ or $d$. The same is true also for the assumptions of the following statement:

Proposition 1. Let $\left(\mathbb{Z}^{2}, p\right)$ be a pretopological space and let $q$ be the quotient pretopology of $p$ on $\mathbb{Z}^{2}$ generated by a surjection $e: \mathbb{Z}^{2} \rightarrow \mathbb{Z}^{2}$. Let e have the property that $e^{-1}(\{y\})$ is connected for every point $y \in \mathbb{Z}^{2}$ and let $D \subseteq \mathbb{Z}^{2}$ be a simple closed curve in $\left(\mathbb{Z}^{2}, q\right)$. Then $D$ is a Jordan curve in $\left(\mathbb{Z}^{2}, q\right)$ if the following two conditions are fulfilled:

(1) There is a Jordan curve $C$ in $\left(\mathbb{Z}^{2}, p\right)$ such that $e(C)=D$.

(2) $C_{i}-e^{-1}(D)$ is nonempty and connected in $\left(\mathbb{Z}^{2}, p\right)$ for $i=1,2$ where $C_{1}$ and $C_{2}$ are the two components of $\mathbb{Z}^{2}-C$.

Proof. Let the conditions of the statement be fulfilled and put $C_{1}^{\prime}=C_{1}-e^{-1}(D)$ and $C_{2}^{\prime}=C_{2}-e^{-1}(D)$. We clearly have $e\left(C_{1}\right) \cap e\left(C_{2}\right)=\emptyset$ (because, otherwise, there is a point $y \in e\left(C_{1}\right) \cap e\left(C_{2}\right)$, which means that $e^{-1}(\{z\}) \cap C_{1} \neq \emptyset \neq$ $e^{-1}(\{z\}) \cap C_{2}$ - this is a contradiction as $e^{-1}(\{z\})$ is connected). Therefore, $e\left(C_{1}^{\prime}\right) \cap e\left(C_{2}^{\prime}\right)=\emptyset$. This yields $C_{i}^{\prime}=e^{-1}\left(e\left(C_{i}^{\prime}\right)\right)$ for $i=1,2$, hence $e\left(C_{i}^{\prime}\right)$ is connected for $i=1,2$ by Lemma 1 . Suppose that $\mathbb{Z}^{2}-D$ is connected. Then $e^{-1}\left(\mathbb{Z}^{2}-D\right)=C_{1}^{\prime} \cup C_{2}^{\prime}$ is connected by Lemma 1 . This is a contradiction because $\emptyset \neq C_{i}^{\prime} \subseteq C_{i}$ for $i=1,2, C_{1}$ and $C_{2}$ are disjoint and $C_{1} \cup C_{2}$ is not connected. Therefore, $\mathbb{Z}^{2}-D=e\left(C_{1}^{\prime}\right) \cup e\left(C_{2}^{\prime}\right)$ is not connected and, consequently, $e\left(C_{1}^{\prime}\right)$ and $e\left(C_{2}^{\prime}\right)$ are components of $\mathbb{Z}^{2}-D$.

The following result is proved in [15]:

Proposition 2. Every cycle in the graph (with the vertex set $\mathbb{Z}^{2}$ ) a portion of which is shown in the following figure is a Jordan curve in $\left(\mathbb{Z}^{2}, w\right)$ :

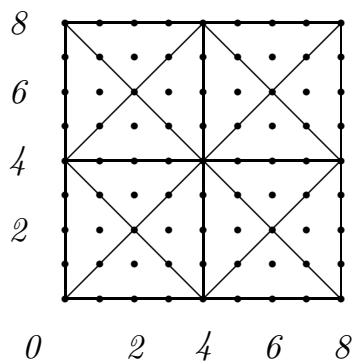


Using Propositions 1-2 and Theorems 1-4, we may identify Jordan curves among the simple closed curves in the Khalimsky and Marcus-Wyse planes, in $\left(\mathbb{Z}^{2}, v\right)$ and in $\left(\mathbb{Z}^{2}, u\right)$. This will be done in the following four Theorems which can be proved in much the same way and, therefore, we will present only the proof of the last of them.

Theorem 5. Let $D$ be a simple closed curve in the Khalimsky plane such that every point $z \in D$ with both coordinates odd satisfies $A_{4}(z) \cap D=\emptyset$. Then $D$ is a Jordan curve in the Khalimsky plane.

Theorem 6. Let $D$ be a cycle in the connectedness graph of the Marcus-Wyse topology such that there exists a point $(x, y) \in \mathbb{Z}^{2}$ with $(x+2 k, y+2 l) \notin D$ whenever $k, l \in \mathbb{Z}$. Then $D$ is a Jordan curve in the Marcus-Wyse plane.

Theorem 7. Let $D$ be a simple closed curve in $\left(\mathbb{Z}^{2}, v\right)$ such that every pair of different points $z_{1}, z_{2} \in D$ with both coordinates even satisfies $A_{4}\left(z_{1}\right) \cap A_{4}\left(z_{2}\right) \subseteq$ $D$. Then $D$ is a Jordan curve in $\left(\mathbb{Z}^{2}, v\right)$.

Corollary 1. Every cycle in the graph a portion of which is shown in the following figure is a Jordan curve in $\left(\mathbb{Z}^{2}, v\right)$.

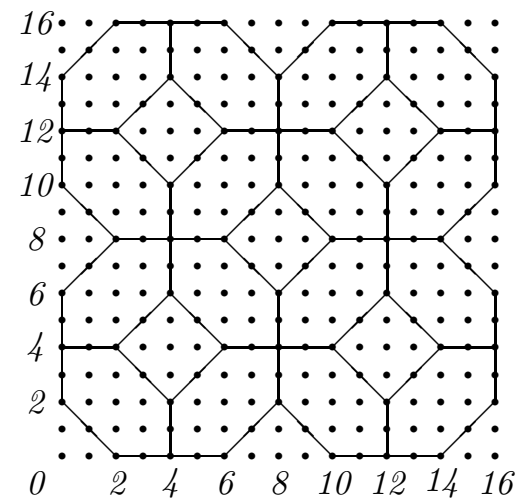

Theorem 8. Every simple closed curve $D$ in $\left(\mathbb{Z}^{2}, u\right)$ which is a cycle in the graph a portion of which is shown in the following figure is a Jordan curve in $\left(\mathbb{Z}^{2}, u\right)$ :

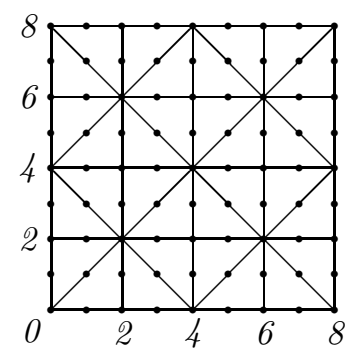


Proof. Let $D$ be a simple closed curve in $\left(\mathbb{Z}^{2}, u\right)$ which is a cycle in the graph a portion of which is shown in the above figure. By Theorem $4, u$ is the quotient pretopology of $w$ generated by $d$. It immediately follows from the definition of $d$ that there exists a unique cycle $C$ in the graph from Proposition 2 such that $d(C)=D$. By Proposition $2, C$ is a Jordan curve in $\left(\mathbb{Z}^{2}, w\right)$. Let $C_{1}, C_{2}$ be the two components of $\mathbb{Z}^{2}-C$ and put $C_{i}^{\prime}=C_{i}-d^{-1}(D)$ for $i=1,2$. Let $(x, y) \in D$ be a point and write $d^{-1}(x, y)$ briefly instead of $d^{-1}(\{(x, y)\})$. Clearly, $d^{-1}(x, y) \nsubseteq \subseteq C$ if and only if both $x$ and $y$ are odd $\left(d^{-1}(x, y)\right.$ is a singleton if $x$ or $y$ is even). Thus, let $(x, y) \in D$ be a point with both $x$ and $y$ odd. Then one of the following two cases occurs:

(1) $(x, y)=(2 k+2 l+1,2 k-2 l+1)$ for some $k, l \in \mathbb{Z}$. Then $d^{-1}(x, y)=$ $A_{4}(4 k, 4 l+2)$, hence $C \cap d^{-1}(x, y)=\{(4 k, 4 l+2+i), i \in\{-1,0,1\}\}$. Therefore, there is $i \in\{1,2\}$ such that $(4 k-1,4 l+2) \in C_{i}-C_{i}^{\prime}$ and $(4 k+1,4 l+2) \in$ $C_{3-i}-C_{3-i}^{\prime}$ while $(4 k-2,4 l+2) \in C_{i}^{\prime}$ and $(4 k+2,4 l+2) \in C_{3-i}^{\prime}, i \in\{1,2\}$. The points $(4 k-1,4 l+1)$ and $(4 k-1,4 l+3)$ are the only points that belong to $C_{i}$ and are adjacent (in the connectedness graph of $\left.w\right)$ to $(4 k-1,4 l+2)$. But both of these points are adjacent also to $(4 k-2,4 l+2) \in C_{i}^{\prime}$. Thus, $C_{i}-\{(4 k-$ $1,4 l+2)\}$ is connected and, by similar arguments, also $C_{3-i}-\{(4 k+1,4 l+2)\}$ is connected.

(2) $(x, y)=(2 k+2 l+1,2 l-2 k-1)$ for some $k, l \in \mathbb{Z}$. Then $d^{-1}(x, y)=A_{4}(4 k+$ $2,4 l)$, hence $C \cap d^{-1}(x, y)=\{(4 k+2+i, 4 l), i \in\{-1,0,1\}\}$. Now, analogously to (1), we can easily show that there is $i \in\{1,2\}$ such that $C_{i}-\{(4 k+2,4 l-1)\}$ and $C_{3-i}-\{(4 k+2,4 l+1)\}$ are connected.

It follows that $C_{1}^{\prime}=d^{-1}\left(d\left(C_{1}^{\prime}\right)\right)$ and $C_{2}^{\prime}=d^{-1}\left(d\left(C_{2}^{\prime}\right)\right)$ are connected. Therefore, $D$ is a Jordan curve in $\left(\mathbb{Z}^{2}, u\right)$ by Proposition 1 .

Remark 1. a) The procedure based on applying Propositions 1 and 2 and used to prove Theorem 8 does not lead to the classical result that, in the Khalimsky plane, any simple closed curve having at least four points is a Jordan curve (Khalimsky, Kopperman and Meyer [5]). For example, if $D$ is a simple closed curve in $\left(\mathbb{Z}^{2}, t\right)$ (having at least four points) which turns at a point with both coordinates odd at the angle $\frac{3 \pi}{4}$, then none of the Jordan curves $C$ in $\left(\mathbb{Z}^{2}, w\right)$ identified in Theorem 5 fulfills $f(C)=D$. Theorem 5 is weaker than the classical result. As for the Marcus-Wyse topology, a Jordan curve theorem different from Theorem 6 was proved in [7].

b) The previous four Jordan curve theorems build on the Jordan curve theorem for $\left(\mathbb{Z}^{2}, w\right)$ given in Proposition 2. Analogously, building on further Jordan curve theorems for $\left(\mathbb{Z}^{2}, w\right)$, we may obtain further Jordan curve theorems in the three topological spaces and the closure space that are quotients of $\left(\mathbb{Z}^{2}, w\right)$. For example, we may build on the following results from [15]: Every cycle in each of the five graphs (with the vertex set $\mathbb{Z}^{2}$ ) portions of which are shown in the following figures is a Jordan curve in $\left(\mathbb{Z}^{2}, w\right)$. 

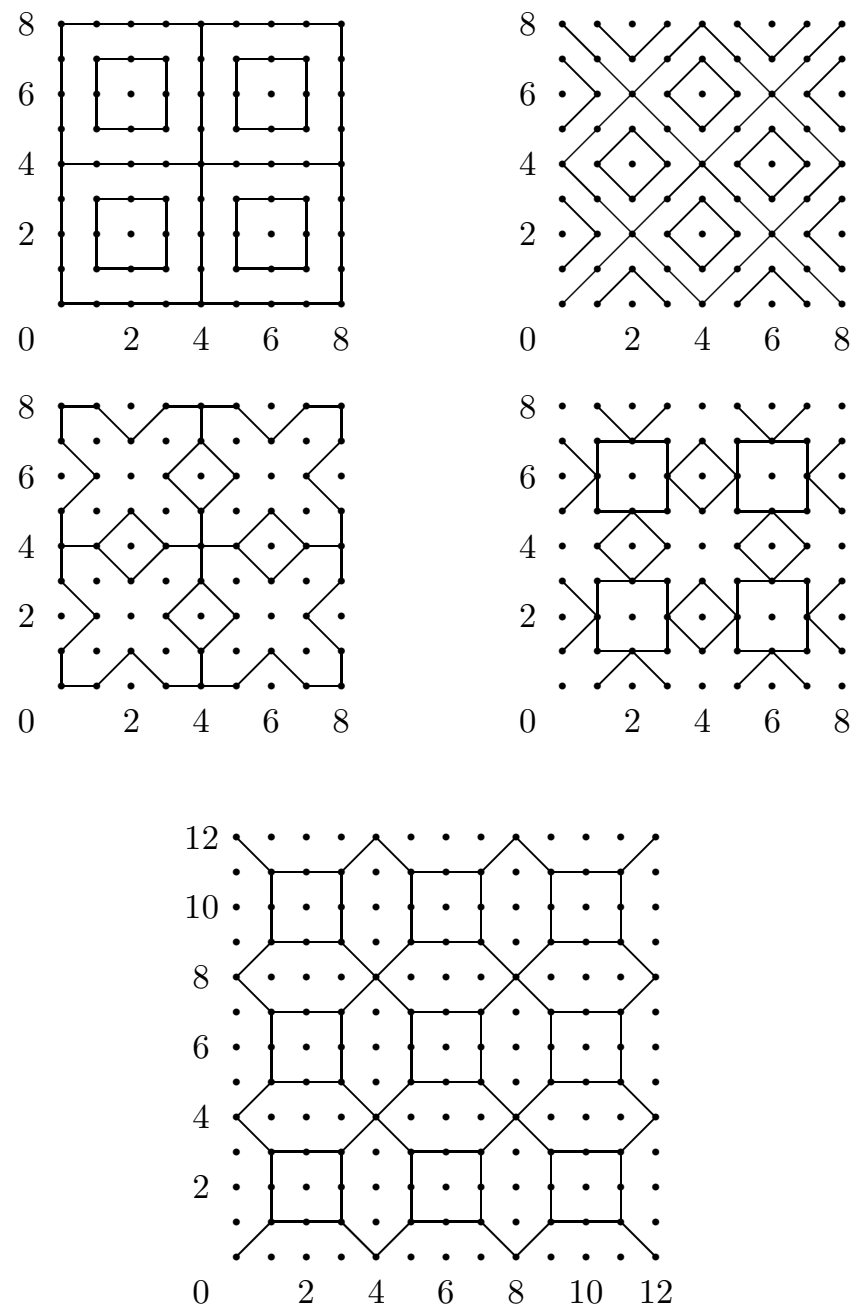

Conclusion. It is well known that, in computer imagery, Jordan curves play an important role because they represent boundaries of regions of digital images. It is therefore useful to work with a connectedness structure on $\mathbb{Z}^{2}$ possessing a considerable variety of Jordan curves. We discuss four such structures, namely topologies $w, t, s, v$ and a pretopology $u$. The results obtained show that these may be used as background structures to solve problems of digital image processing, especially those closely related to boundaries (image data compression, pattern recognition, boundary detection and contour filling, etc.).

Acknowledgement. The author acknowledges partial support from Ministry of Education of the Czech Republic, research plan no. MSM0021630518. 


\section{References}

1. Čech, E.: Topological Spaces (Revised by Z. Frolík and M. Katětov). Academia, Prague (1966)

2. Eckhardt, U., Latecki, L.J.: Topologies for the digital spaces $\mathbb{Z}^{2}$ and $\mathbb{Z}^{3}$. Comput. Vision Image Understanding 90, 295-312 (2003)

3. Engelking, R.: General Topology. Państwowe Wydawnictwo Naukowe, Warszawa (1977)

4. Khalimsky, E.D.: On topologies of generalized segments. Soviet Math. Dokl. 10, 1508-1511 (1999)

5. Khalimsky, E.D., Kopperman, R., Meyer, P.R.: Computer graphics and connected topologies on finite ordered sets. Topology Appl. 36, 1-17 (1990)

6. Khalimsky, E.D., Kopperman, R., Meyer, P.R.: Boundaries in digital planes. Jour. of Appl. Math. and Stoch. Anal. 3, 27-55 (1990)

7. Kiselman, C.O.: Digital Jordan curve theorems. In: Nyström, I., Sanniti di Baja, G., Borgefors, G. (eds.) DGCI 2000. LNCS, vol. 1953, pp. 46-56. Springer, Heidelberg (2000)

8. Kong, T.Y., Kopperman, R., Meyer, P.R.: A topological approach to digital topology. Amer. Math. Monthly 98, 902-917 (1991)

9. Kopperman, R., Meyer, P.R., Wilson, R.G.: A Jordan surface theorem for threedimensional digital spaces. Discr. and Comput. Geom. 6, 155-161 (1991)

10. Marcus, D., et al.: A special topology for the integers (Problem 5712). Amer. Math. Monthly 77, 1119 (1970)

11. Rosenfeld, A.: Digital topology. Amer. Math. Monthly 86, 621-630 (1979)

12. Rosenfeld, A.: Picture Languages. Academic Press, New York (1979)

13. Šlapal, J.: Closure operations for digital topology. Theor. Comp. Sci. 305, 457-471 (2003)

14. Šlapal, J.: A digital analogue of the Jordan curve theorem. Discr. Appl. Math. 139, 231-251 (2004)

15. Šlapal, J.: Digital Jordan curves. Top. Appl. 153, 3255-3264 (2006)

16. Šlapal, J.: A quotient-universal digital topology. Theor. Comp. Sci. 405, 164-175 (2008) 